\title{
A Head Motion Measurement System Suitable for Emission Computed Tomography
}

\author{
Seth R. Goldstein,* Margaret E. Daube-Witherspoon, Member, IEEE, Michael V. Green, and Alec Eidsath
}

\begin{abstract}
Subject motion during brain imaging studies can adversely affect the images through loss of resolution and other artifacts related to movement. We have developed and tested a device to measure head motion externally in real-time during emission computed tomographic (ECT) brain imaging studies, to be used eventually to correct ECT data for that motion. The system is based on optical triangulation of three miniature lights affixed to the patient's head and viewed by two position-sensitive detectors. The computer-controlled device converts the three sets of lamp positions into rotational and translational coordinates every 0.7 seconds. When compared against a mechanical test fixture, the optical system was found to be linear and accurate with minimal crosstalk between the coordinates. In a study of two subjects, comparing the angular motions measured by the optical device and a commercially available electromagnetic motion detector, the two systems agreed well, with an root mean square (rms) difference of less than $0.6^{\circ}$ for all rotations.
\end{abstract}

Index Terms-Brain imaging, motion correction, PET, SPECT.

\section{INTRODUCTION}

$\mathbf{E}$ MISSION computed tomography (ECT) brain studies can require from 30 minutes to several hours to complete, during which time the subject must lie still. The usual approach to this problem is to restrain the subject's head to the greatest degree possible consistent with patient comfort and general practicality. Even with substantial head restraint, however, some amount of head motion is inevitable, especially in less cooperative subjects [1], [2]. This residual motion can adversely affect the ECT study in a number of ways: 1) loss of resolution in an individual scan; 2) misalignment of scans in a sequence that are assumed to be aligned; 3) motion artifacts, especially in single photon emission computed tomography (SPECT) studies, in which motion during the rotation of the cameras will lead to an inconsistent data set.

Manuscript received September 28, 1995; revised September 30, 1996. The Associate Editor responsible for coordinating the review of this paper and recommending its publication was C. R. Meyer. Asterisk indicates corresponding author.

*S. R. Goldstein is with the Biomedical Engineering and Instrumentation Program, National Center for Research Resources, National Institutes of Health, Bldg. 13, Rm. 3W13, Bethesda, MD 20892 USA (e-mail: sethg@boxs.nih.gov).

M. E. Daube-Witherspoon is with the Positron Emission Tomography Department, Clinical Center, National Institutes of Health, Bethesda, MD 20892 USA

M. V. Green is with the Nuclear Medicine Department, Clinical Center, National Institutes of Health, Bethesda, MD 20892 USA.

A. Eidsath is with Biomedical Engineering and Instrumentation Program, National Center for Research Resources, National Institutes of Health, Bethesda, MD 20892 USA

Publisher Item Identifier S 0278-0062(97)00982-8.
A number of methods have been reported to correct for head motion, based upon the image data themselves [3]-[7]. These methods suffer from the common disadvantage of depending upon the quality of the scan data-including resolution, sampling, and noise characteristics-as well as the need for the activity distribution not to change significantly over the course of the study.

Because of these disadvantages, an external real-time measurement of head motion was deemed preferable. There are several devices to measure subject motion that are potentially applicable to ECT brain studies. Electromagnetic systems, such as the Spacetracker (Polhemus Navigational Sciences, Colchester, VT) and the Bird (Ascension Technology Corporation, Burlington, VT), suffer from interference from eddy currents induced in the metal in the gantry that seriously compromises the accuracy of the motion measurement. Acoustical devices, such as the GP8-3D Sonic Digitizer (Science Accessories Corporation, Stratford, CT), produce an audible signal that may be unacceptable for brain imaging, especially for activation studies. Optical/videometric devices such as the Optotrak (Northern Digital, Waterloo, Ont., Canada) or the systems based upon charge coupled device (CCD) cameras described by Dubey et al. [8], Picard and Thompson [9], and Menke et al. [10] are also potentially applicable for measuring head motion and meet many of the design objectives (see below), although the attachment of the reflectors to the subject for the last system is rather invasive.

The design of a system that avoids all of the drawbacks of the existing devices while retaining the necessary accuracy and reliability was undertaken. The design objectives for the proposed system were as follows:

1) accuracy $\left(<0.5-\mathrm{mm}\right.$ translation, $<0.5^{\circ}$ rotation);

2) sampling rate of at least $1 \mathrm{~Hz}$;

3) minimal subject disturbance;

4) user-friendliness with easy and fast setup and usage;

5) no impact on ECT scanner operation;

6) insensitivity to ECT scanner operation.

The accuracy and sampling requirements were based upon measurements of head motion taken in normal and schizophrenic subjects [1], as well as the resolution and sampling of current-generation ECT scanners. Because the system was intended for use during brain scans, it was also desired to minimize the invasiveness of any attachments to the subject.

The device was initially designed for use in a brain positron emission tomography (PET) scanner (model PC2048-15B, Scanditronix, Uppsala, Sweden) which imposed some additional constraints on the design. These included the following: 


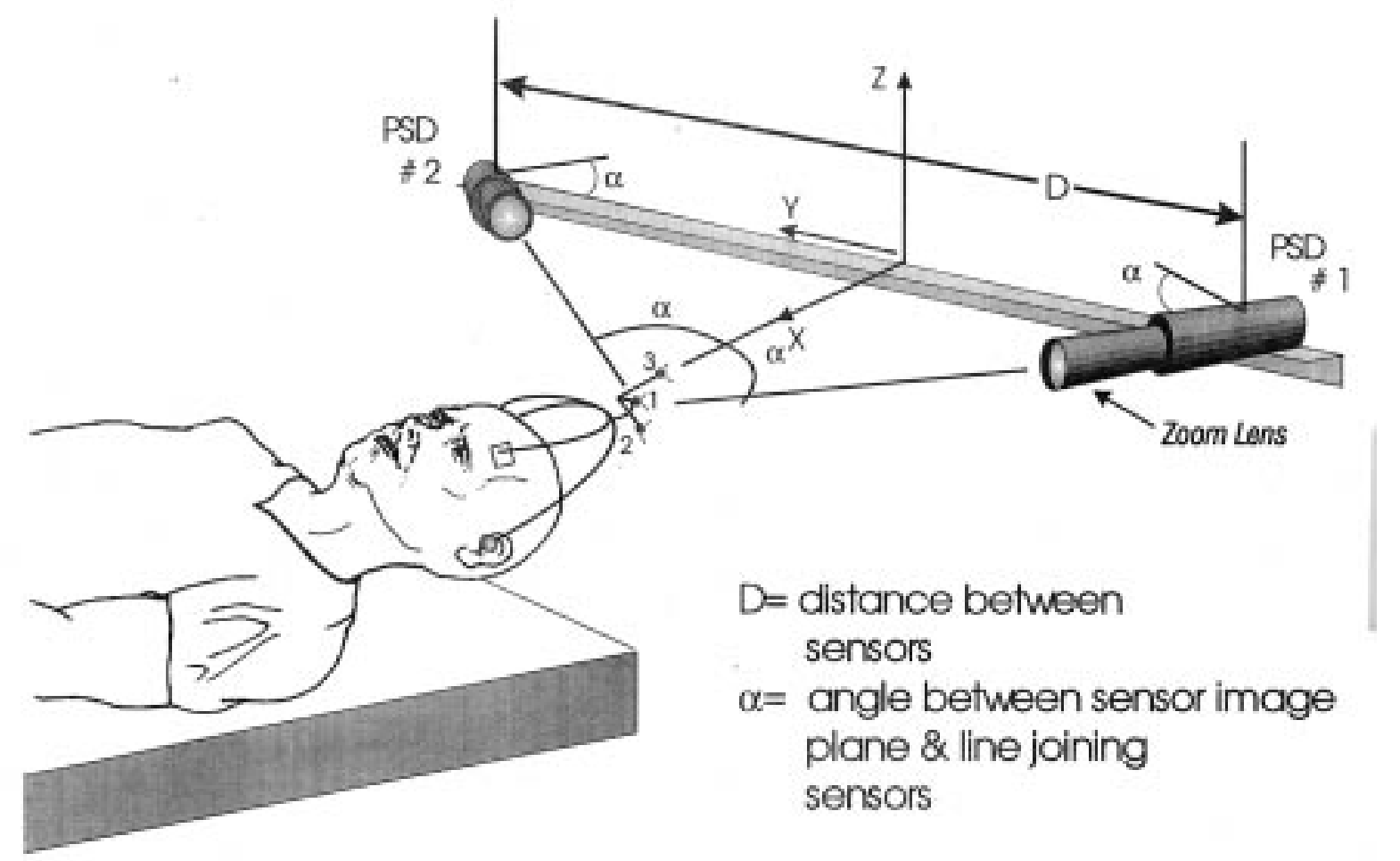

Fig. 1. Schematic illustration of OMD system. The 3-D coordinates of each of three blinking lights of a triad attached to the head are calculated from the two sets of $x-y$ coordinates in the image planes of two PSD's. These sensors provide voltages proportional to the position of the centroid of light falling on their image planes. The lenses/sensors are external to the scanner and are focused at the nominal location of the triad when it is mounted on a subject in the PET scanner.

1) limited space both inside the gantry (patient aperture $=$ $27 \mathrm{~cm}$ ) and around the scanner (less than $60 \mathrm{~cm}$ between the rear of the scanner and the wall);

2) further reduced access to the head, especially from the front, due to clinical and/or experimental apparatus;

3) numerous metallic parts in the PET scanner (which obviated the possibility of using radio frequency (RF)based detectors).

This paper describes an optical motion detector (OMD) to detect and quantify head motion and presents experimental results for a mechanical test fixture that validate the accuracy and linearity of the system. The head attachment mechanism is also described, and experimental results comparing the OMD and a commercial motion detection system in subjects are presented.

\section{SYSTEM DESCRIPTION}

\section{A. Overview}

The OMD is shown schematically in Fig. 1. A triad of miniature incandescent lights is attached to the subject's head by means of a lightweight tubular framework. The threedimensional (3-D) position of each light is determined by triangulation, based upon the two-dimensional (2-D) information from each of two electrooptical position sensitive detectors (PSD's), as each light is blinked sequentially under conditions of low ambient lighting. The translational motions of the triad are determined from changes in the coordinates of the center lamp. The three sets of 3-D coordinates for the three lights are converted to the Euler angles of the lamp triad assembly to determine the rotational motions of the head. The blinking of the lights, data acquisition from the two PSD's, coordinate computations, and data recording and storage are all under computer control. The various subsystems are described in greater detail below. The rationale for and implications of the selection of incandescent lamps as a light source is treated in Section V.

\section{B. Lamp Assembly and Its Attachment to the Head}

A photograph of the lamp assembly is shown in Fig. 2. Three tungsten microlamps (ML7119 Microlamps, Batavia, IL) are located at the vertices of a $45^{\circ}$ right triangle which is $7.6 \mathrm{~cm}$ on a side. The lamps are $2 \mathrm{~mm}$ in diameter by 8 $\mathrm{mm}$ in length, have a filament that is $\sim 1 \mathrm{~mm}^{3}$, and draw 0.1 A from an isolated 5-V power supply. Each lamp is recessed $1 \mathrm{~mm}$ inside a $2.1-\mathrm{mm}$ diameter steel tube to reduce stray illumination. The tube has two 1-mm wide by $2-\mathrm{mm}$ long axial notches in the plane of the two sensors in order to enhance light to the sensors over light to other (reflective) objects. The sides of the triangular assembly are made of hollow stainless steel tubing (1-mm diameter) that is attached to a plastic ball (1$\mathrm{cm}$ diameter). The ball, in turn, is held in a socket affixed to a thin tubular framework that attaches to the subject's head. The triad orientation can be roughly aligned with the scanner axes by maneuvering the ball in its socket and then tightening the assembly. Lightweight flexible cables connect the three lamps to an electrical control box that sequentially blinks each lamp under computer control. 


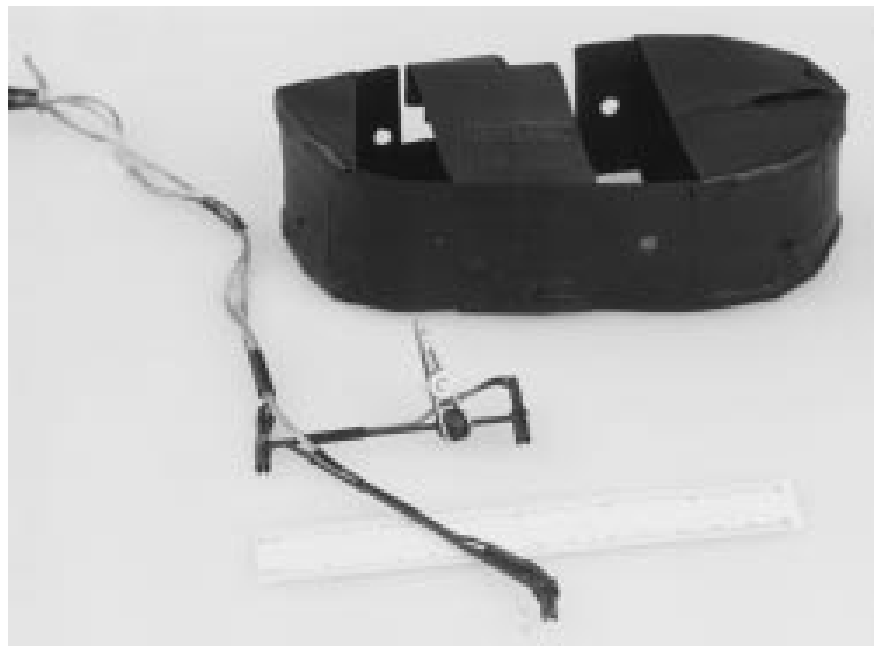

Fig. 2. Lamp triad assembly. The lightweight tubular assembly holds short, notched, blackened tubes in which miniature bulbs are slightly recessed to minimize stray light. An additional external shield (shown detached from the support tube) is used to reduce stray light (e.g., from reflections off of the inner surface of the gantry) further. A miniature lamp is also shown.

Shown adjacent to the triad in Fig. 2 is the lightweight auxiliary light shield that fits onto the triad assembly. This blackened shield prevents the sensors from detecting stray light reflected off of adjacent surfaces (e.g., the inner bore of the gantry), while allowing light from the lamps to shine directly onto the sensors (see Section V).

Fig. 3 is a photograph of the complete triad and supporting framework assembly mounted on a person's head. The framework consists of two U-shaped stainless steel tubes $(\sim 1-\mathrm{mm}$ diameter), hinged to each other. The ends of one tube have soft plastic hemispheres that fit into the outer ear openings, while the ends of the other tube plug into electrode patches attached to the subject's temples for the purpose of stabilizing the attachment. Both U-shaped tubes are preloaded against the head with elastic bands to ensure that their terminations are in good contact with the ears and temples. The tubes and their end fixtures are adjustable in length and orientation to accommodate different head sizes.

\section{Measurement of 3-D Lamp Positions}

As shown in Fig. 1, two zoom lenses in c-mount housings (f\#/1.2, 12.5-75-mm focal length set at $28 \mathrm{~mm}$ ), separated by a distance $D(D=1.25 \mathrm{~m})$ and tilted at an angle $\alpha$ are mounted on a rail so that their optical axes are in the same horizontal plane. We selected $\alpha=38.5^{\circ}$, based on the shape of the scanner bore, the depth of the triad from the lenses, and the desire to be as close to $45^{\circ}$ as possible based on an error analysis of the triangulation procedure. The rail is stably mounted (e.g., to the wall behind the scanner) in a position such that the intersection of the optical axes of the two lenses is at the nominal location of the lamp triad when it is mounted on a subject lying in the scanner ( $\sim 0.9 \mathrm{~m}$ from each lens). Error analysis has been done which indicates that small deviations from this ideal geometry are not of practical importance.

A PSD (model OT-3210, On-Trak Photonics, Lake Forest, CA) is located at the focal plane of each lens and is connected

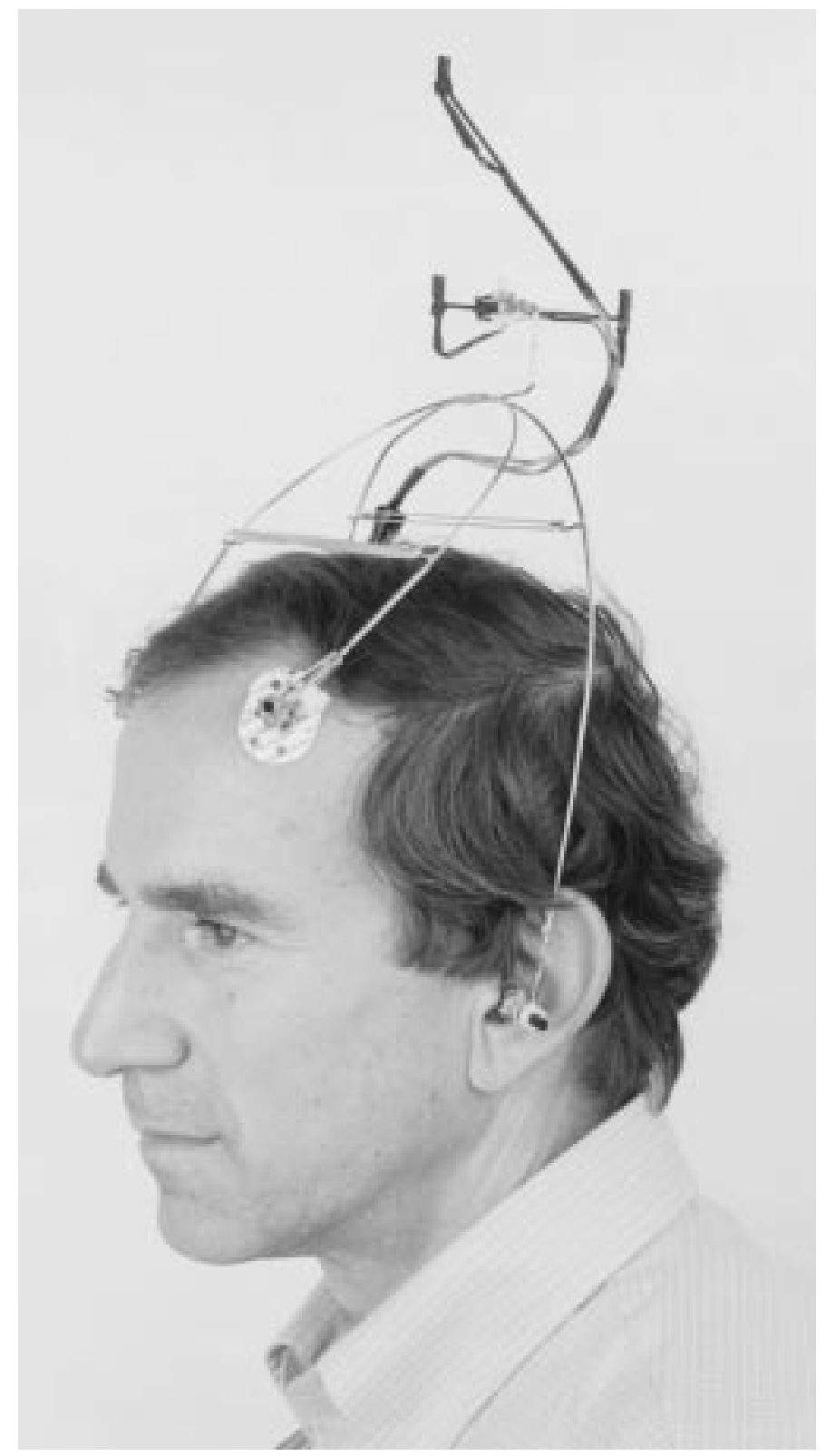

Fig. 3. Attachment of lamp assembly to a subject's head by the tubular support framework. Standard adhesive electrode patches placed at the temples provide mounting points to stabilize the assembly after the plastic hemispheres have been lightly inserted in the ear openings.

to an analog electronic signal processor (On-Trak Photonics, model OT-300). These silicon sensor devices have a $1-\mathrm{cm}$ by $1-\mathrm{cm}$ active area and two orthogonal pairs of electrodes that pass the current created when light strikes the photosensitive surface. Since the resistance between the point of current generation on the sensor surface and the various electrodes is a function of the position of the current generation, the difference between the currents in each pair of electrodes is directly proportional to the position of the centroid of the light falling on the sensor. The effect of illumination intensity on the position measurement is nominally eliminated by dividing each current difference by the sum of the currents from the two electrode pairs. These devices have a typical accuracy of $0.1 \%$ over $90 \%$ of the active area. As the lamps are sequentially 
turned on and off, the vertical and horizontal coordinates in the image plane of each sensor are thus determined for each lamp. A complete set of raw data (three sets of $x$ - $y$ light positions for both sensors) is recorded every 0.7 seconds. The rate-limiting step is the approximately $0.2 \mathrm{~s}$ it takes for each tungsten bulb to turn on and reach a steady-state light intensity. (The divider in the commercially supplied electronics is not high bandwidth and does not provide perfect compensation on a millisecond time scale; this can be corrected in a straightforward manner but was not within the scope of this work.)

Although the system does not need complete darkness for accurate operation, it is desirable that the ambient light level be small compared with that from the triad, since the measured current results from the centroid of all of the light hitting the sensor. ECT studies are typically performed under these conditions and we do not anticipate that our required ambient light levels will be a limitation. The On-Trak Photonics signal processor does not have the capability to subtract out background light prior to centroid calculation. However, the procedure for measuring the position of the reference light (discussed in Section II-F) effectively compensates for low levels of ambient light (assuming they are roughly comparable to the light levels during patient studies). Changes in the ambient light (e.g., from opening of doors or visual activation equipment) are also potential sources of error, although their impact on the motion correction can be made small through careful shielding of the detectors from these extra light sources and by insuring that their magnitude is small compared to the lamp intensity.

\section{Computer Control}

A personal computer (Macintosh Quadra 650) using commercially available software (LabView National Instruments, Austin, TX) with an input/output (I/O) board (National Instruments Lab NB) performs data acquisition and storage and controls the blinking of the lights. In addition, the computer performs all mathematical calculations, provides graphical and numerical outputs of the data in real-time, and monitors the lamps' light levels and provides a warning if they are too low or change too much. Special routines are also provided to measure the position of a reference light at startup and to calibrate the system.

\section{E. Conversion of Sensor Coordinates into Position and Orientation Information}

The two sets of PSD horizontal and vertical coordinates for each lamp are converted into 3-D coordinates in the coordinate system of the scanner in order to determine the three translation and the three rotation coordinates of the motion. The geometry of the system is shown in Fig. 4, where the vertical axis $(Z)$ and the two sensor image planes are perpendicular to the page. It is assumed that the optical axes of the lens/sensor both lie in the plane of the figure. Also, the sensors are rotationally adjusted so that their vertical axes are parallel to the $Z$ axis. For simplicity, the demagnification and image reversal caused by the zoom lenses are omitted from the figure and following discussion.
The three sets of 3-D light coordinates, $(X, Y, Z)_{p}$, for light $p(p=1-3)$, are derived from the sensor coordinates, $x_{i p}, y_{i p}$ for sensor $i(i=1,2)$ by the following equations:

$$
\begin{aligned}
X_{p} & =\frac{\left(x_{2 p}-x_{1 p}\right)}{2 \sin \alpha} R_{p} S+X_{r} \\
Y_{p} & =\frac{\left(x_{1 p}+x_{2 p}\right)}{2 \cos \alpha} R_{p} S+Y_{r} \\
Z_{p} & =\frac{\left(y_{1 p}+y_{2 p}\right)}{2} R_{p} S+Z_{r}
\end{aligned}
$$

where the subscript $r$ refers to the reference light position, described below, and $R_{p}$ corrects for the difference in distance to the sensors between lights $p=1,2$ and the front light ( $p=$ 3) (an effect omitted from Fig. 4). Since lights one and two are roughly equidistant from the detectors, $R_{1}=R_{2}=1.0$. Lamp three is closer to the detectors, and the value of $R_{3}$ is $0.97 . S$ is a scale factor determined during system calibration (discussed below) which is performed with the triad in approximately the same position as when it is on a subject.

The geometry of the triad and the associated Euler angles are shown in Fig. 5. Leg 1-3 of the triad is nominally parallel to the scanner bore, pointing toward the lenses. (For ease of visualization, lamp two is shown extending above lamp one. In actual practice, and in agreement with the setup shown in Fig. 1, lamp two extends below rather than above lamp one.) The equations for computing the Euler angles from the three sets of 3-D coordinates $(X, Y, Z)_{p}$ of each lamp were derived using a standard rigid-body transformation [1] (see Fig. 5). The final result (with the sign of $\Psi$ reversed from the setup shown in Fig. 5) is

$$
\begin{aligned}
\operatorname{Azimuth}(\Phi) & =\tan ^{-1}\left(\frac{Y_{3}-Y_{1}}{X_{3}-X_{1}}\right) \\
\operatorname{Elevation}(\Theta) & =-\tan ^{-1}\left(\frac{Z_{3}-Z_{1}}{\sqrt{\left(X_{3}-X_{1}\right)^{2}+\left(Y_{3}-Y_{1}\right)^{2}}}\right) \\
\operatorname{Roll}(\Psi) & =\tan ^{-1}\left(\frac{\left(Y_{2}-Y_{1}\right) \cos \Theta}{\left(Z_{2}-Z_{1}\right) \cos \Phi}-\sin \Theta \tan \Phi\right) .
\end{aligned}
$$

The triad's location with respect to the reference lamp is specified by the central lamp (\#1), so that the translational coordinates are given by

$$
\begin{aligned}
\text { Axial } & =X_{1} \\
\text { Lateral } & =Y_{1} \\
\text { Vertical } & =Z_{1} .
\end{aligned}
$$

The three Euler angles plus the three translational coordinates of the triad are sufficient to specify completely the head motions during a scan with respect to the PET scanner coordinates if the triad is assumed to move with the brain.

\section{F. Calibration, Reference Position, and Alignment}

The device requires calibration to account for the effects of the lens focal length, sensor size, maximum signal output, and analog-to-digital (A/D) converter full scale. During calibration, the triad is oriented with its two legs approximately vertical and horizontal and pointed approximately toward the midpoint 


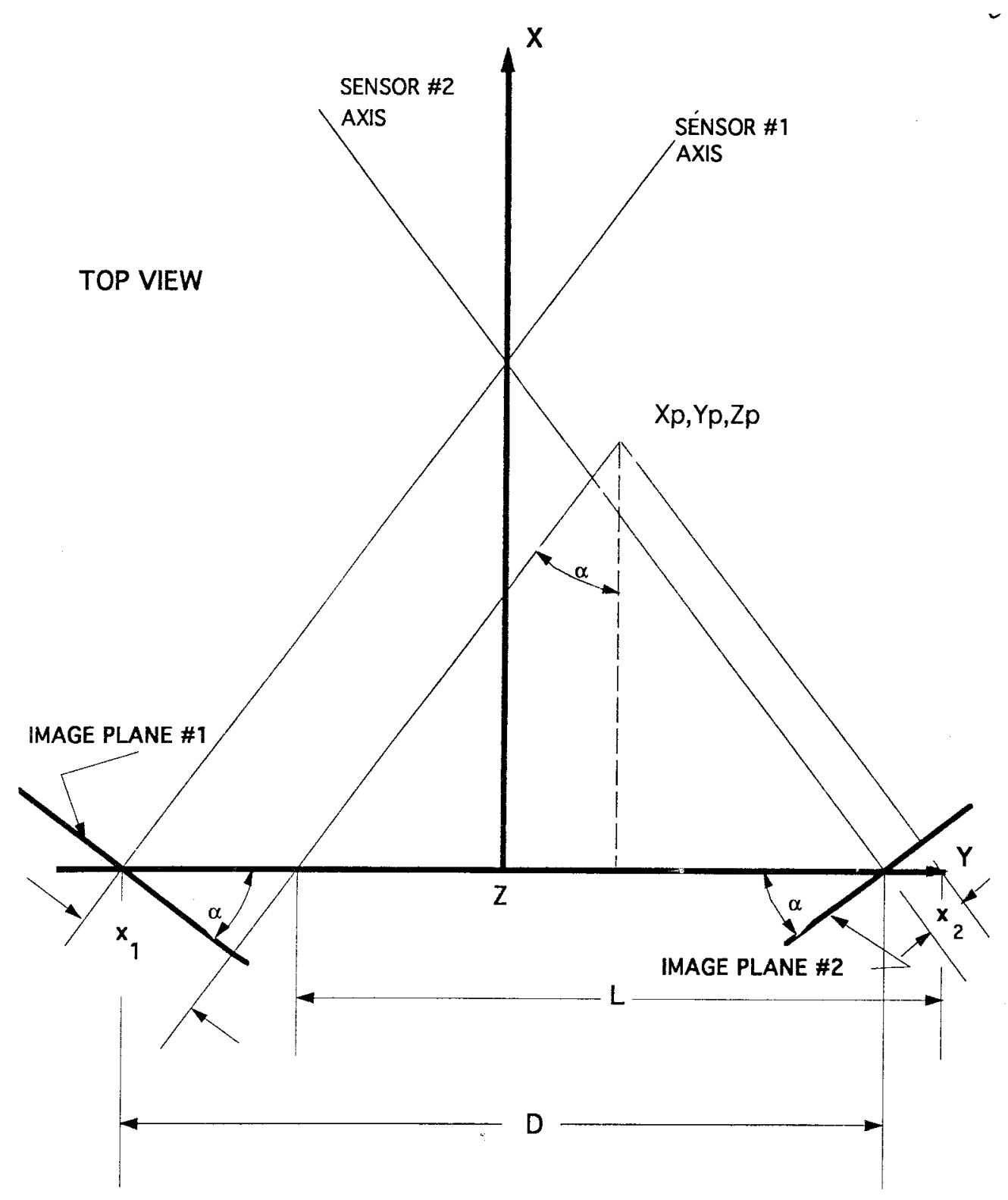

Fig. 4. Plan view showing triangulation geometry. The $X$ axis points into the scanner parallel to the bore centerline in agreement with Fig. 1. The $Y$ axis is parallel to the scanner's horizontal axis, while the $Z$ axis is parallel to the scanner's vertical axis (out of the page). The sensors' $y$ axes are parallel to the $Z$ axis.

between the two sensors. The scale factor, $S$, in (1) is determined from the ratio of the measured and known distances between lights one and two. Although $S$ is determined routinely only from measurements made along the $y$-axis of the PSD, it has been measured to be essentially identical along the $x$-axis, so the same value for $S$ can be used in all three equations in (1).

All coordinates are measured relative to the position of a reference light. The reference light is a fixture that is placed in a repeatable manner at a point that is nominally where the triad would be during head motion measurements. This reference light position has been accurately located with respect to the center of the PET scanner by imaging a triad of capillaries filled with a positron-emitter (this measurement also gives the orientations between the scanner axes and the sensor system).
The 3-D coordinates of the reference light are periodically measured when the system is calibrated; the reference lamp is then moved out of the way so that it will not interfere with the lamp triad during head motion measurements. Thus, all measurements of the triad lights on a subject are of small distances about a known location so the system accuracy does not depend on its being linear over a much larger range.

\section{Materials AND MethodS}

\section{A. Noise and Stability}

The short-term variability (noise) of the system was measured by monitoring the outputs with the lamps held stationary for 60 minutes. This duration was chosen as representative of a typical ECT patient study. The system stability was measured 


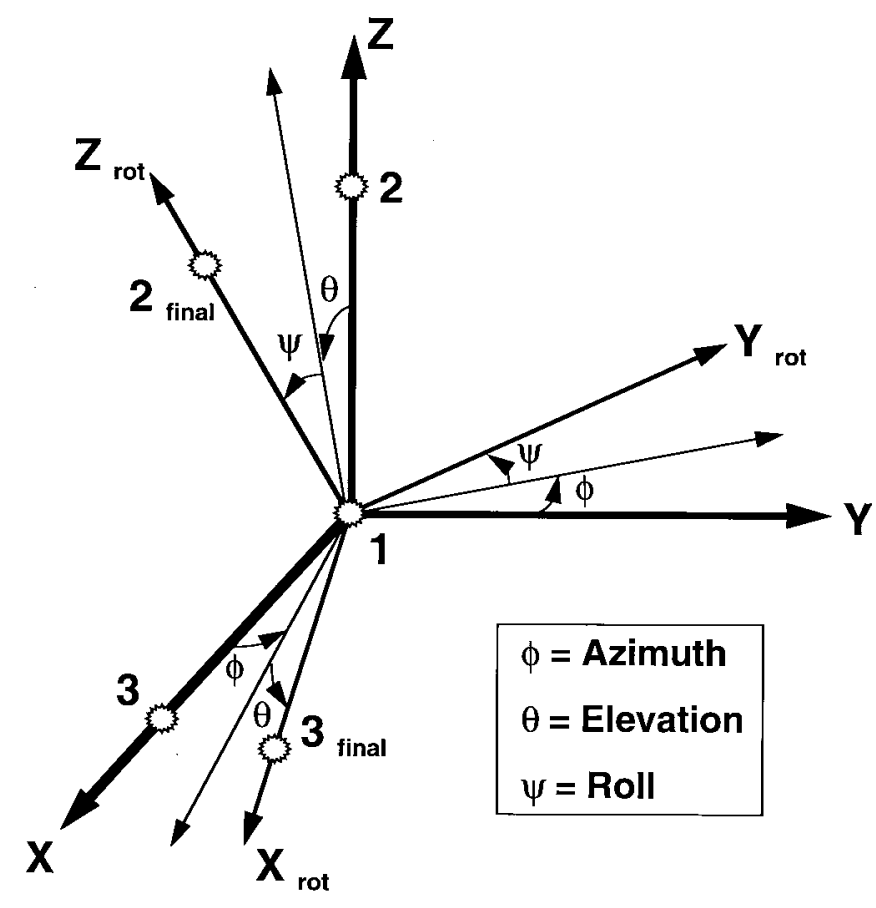

Fig. 5. Geometry of lamp triad and its Euler angles, as viewed looking from the PSD's toward the triad.

by conducting the same test for a period of 16.8 hours. These data were divided into 20-min frames, and the mean and standard deviation of the frames were calculated to assess long-term stability. The system stability was characterized by the maximum and average absolute deviations of the six measured coordinates over the course of the acquisition. To eliminate the effects of changes in ambient light, both tests were conducted in a darkened room.

\section{B. System Linearity, Accuracy, and Crosstalk}

To assess the linearity and accuracy of the system, the lamp triad was mounted inside the gantry of the Scanditronix PC2048-15B brain PET scanner on a special mechanical fixture which produces known, controlled, steady-state positions. This fixture provides separate micrometer adjustments for lateral and vertical translations to within $\pm 0.1 \mathrm{~mm}$, and roll, elevation and azimuth adjustments to within $\pm 0.25^{\circ}$. Axial positions, performed by sliding the entire mechanism against a guide, were accurate to $\sim \pm 0.25 \mathrm{~mm}$. Changes were carried out in all six degrees of freedom (DOF's) independently over a translational range of $\pm 20-30 \mathrm{~mm}$ and an angular range of $\pm 4^{\circ}$. Because stray reflections from nearby surfaces or misalignment of the two sensors can give rise to apparent motions in directions where there is no real motion, this study also assessed the degree of crosstalk in the system as the result of motion in each direction.

Outputs in response to a series of controlled inputs were measured with the ambient illumination held approximately constant at a low level. During controlled translations, all six outputs were monitored in order to assess the crosstalk between the six measured parameters. During rotations, the lateral, vertical, and axial motions were ignored, since the lamps would also translate with a rotation, depending upon the (unknown) distance between the lamps and the axes of rotation, and observed motion in these directions would not provide a meaningful measurement of crosstalk alone. All measurements were performed while the triad was stationary (i.e., after it had been moved to a given position and orientation).

\section{Accuracy in Subjects}

In order to assess the accuracy of the motion detection device and head attachment mechanism in human subjects, the OMD and the Spacetracker, a commercially available electromagnetic position-tracking system, were used simultaneously to measure head motion in two normal volunteers on each of two occasions, for a total of four measurements. The tests were performed in a darkened room away from large metallic objects, in order to avoid measurement artifacts with the RFbased Spacetracker. The two systems were aligned with one another and simultaneously tested first using the previously described mechanical fixture to ensure that their outputs were similar for known inputs. The Spacetracker transducer was then attached to a custom-formed biteplate that was held firmly in place by the subject's teeth; the light triad was attached to the head, as previously described. The subjects first performed a series of small $\left(<10^{\circ}\right)$, controlled angular head movements for 6-7 min They then rested quietly for an additional 10-20 min. One subject performed an additional set of controlled movements after the quiet period, as well. The Spacetracker recorded data approximately every two seconds, while the OMD sampled every $0.7 \mathrm{~s}$.

The motion data from the two systems were compared by interpolating the OMD data to the (longer) time intervals from the Spacetracker. Spikes were seen in both the OMD and Spacetracker data at the times of abrupt changes in orientation as a result of inconsistent data being acquired when the subject moved during a sampling period. Correction was made for these spikes in the data by masking out any points with a first derivative greater than ten times the average first derivative. The rms and maximum absolute differences between the two data sets were calculated for the entire study and for the periods of motion and quiet separately.

\section{RESUlts}

\section{A. Noise and Stability}

The light level at the sensor faceplate is $1.2 \mathrm{~mW}$ from the middle and front lamps and half that amount from the lower lamp, as measured by a radiometer placed at the rear of the zoom lens, at the position of the faceplate of the PSD sensor. This level, which affects the noise in the measurement of motion, is over an order of magnitude larger than the typical background illumination during an ECT study. Ambient light would, therefore, not be expected to affect head motion data significantly.

The levels of system noise were characterized by the standard deviation of the measurements of the six coordinates during the 60-min acquisition. The standard deviations for the 
TABLE I

LINEARITY AND ACCURACY OF OMD

\begin{tabular}{ccc}
\hline Motion & Slope & $\mathrm{R}^{2}$ \\
\hline Axial & $0.983 \pm 0.004$ & 0.99987 \\
Lateral & $0.993 \pm 0.003$ & 0.99993 \\
Vertical & $1.004 \pm 0.002$ & 0.99998 \\
Azimuth & $0.991 \pm 0.009$ & 0.99944 \\
Elevation & $0.904 \pm 0.011$ & 0.99904 \\
Roll & $0.990 \pm 0.002$ & 0.99996 \\
\hline
\end{tabular}

TABLE II

CRosstalk OF OMD

\begin{tabular}{ccccccc}
\hline Motion & Axial $^{*}$ & Lateral $^{*}$ & Vertical $^{*}$ & Azimuth $^{\#}$ & Elcvation $^{\#}$ & Roll $^{\#}$ \\
\hline Axial & --- & 0.14 & 0.42 & NS & 0.26 & NS \\
Lateral & 0.36 & --- & -0.15 & -0.09 & -0.04 & 0.09 \\
Vertical & -0.20 & 0.12 & --- & 0.04 & 0.05 & 0.04 \\
Aximuth & & & & -- & -0.11 & -0.08 \\
Elevation & & & & -0.02 & --- & -0.16 \\
Roll & & & & -0.05 & -0.07 & --- \\
\hline
\end{tabular}

\section{${ }^{*} \mathrm{~mm} / \mathrm{cm}$ input}

\#\% or $\%$ deg input

NS - Less than $0.01 \mathrm{~mm} / \mathrm{cm}$ input or $0.01 \% \mathrm{~cm}$ or $\% \mathrm{deg}$ input

axial, lateral, and vertical directions were $0.092 \mathrm{~mm}, 0.047$ $\mathrm{mm}$, and $0.042 \mathrm{~mm}$, respectively. The standard deviations of the measured roll, elevation, and azimuth angles were $0.034^{\circ}$, $0.047^{\circ}$, and $0.039^{\circ}$, respectively.

The maximum absolute deviations for the axial, lateral, and vertical directions for the longer acquisition were $0.66 \mathrm{~mm}$, $0.32 \mathrm{~mm}$, and $0.44 \mathrm{~mm}$, respectively. The average absolute differences for the same directions were $0.46 \mathrm{~mm}, 0.23 \mathrm{~mm}$, and $0.28 \mathrm{~mm}$. The maximum absolute differences of the measured roll, elevation, and azimuth angles were $0.032^{\circ}$, $0.048^{\circ}$, and $0.107^{\circ}$, respectively. The average absolute deviations for the same angles were $0.007^{\circ}, 0.022^{\circ}$, and $0.073^{\circ}$. The average standard deviations of these data were $0.03 \mathrm{~mm}$ for the translational motions and $0.03^{\circ}$ for the rotations. It was observed that the deviations decreased noticeably after 9 P.M. The maximum absolute differences of the translational motions were reduced to $<0.25 \mathrm{~mm}$ for the remainder of the acquisition $(11.5 \mathrm{~h})$; the maximum absolute deviations for the rotational motions were reduced by a factor of two from the above values.

\section{B. System Linearity, Accuracy, and Crosstalk}

The system linearity and accuracy, as determined by the response of the OMD to known inputs, are presented in the plots of Fig. 6. Typically, five data readings were averaged for each point in these plots. The best-fit line and the line of identity are also shown. The slopes and square correlation coefficient $\left(R^{2}\right)$ of the best-fit lines through the data are summarized in Table I. The results demonstrate that the system is linear. Most of the slopes are within $1 \%$ of unity; the slope for the elevation measurement was only 0.90 . The rms
TABLE III

COMPARISON OF OMD TO SPACETRACKER

\begin{tabular}{llcc}
\hline Period & Motion & rms difference & $\begin{array}{c}\text { Maximum absolute } \\
\text { difference }\end{array}$ \\
\hline \hline ENTIRE & Roll & $0.26^{\circ} \pm 0.09^{\circ}$ & $1.85^{\circ} \pm 0.77^{\circ}$ \\
& Elevation & $0.49^{\circ} \pm 0.13^{\circ}$ & $2.50^{\circ} \pm 0.79^{\circ}$ \\
& Azimuth & $0.29^{\circ} \pm 0.06^{\circ}$ & $1.98^{\circ} \pm 0.49^{\circ}$ \\
\hline \hline QUIET & Roll & $0.17^{\circ} \pm 0.07^{\circ}$ & $0.97^{\circ} \pm 0.87^{\circ}$ \\
& Elevation & $0.42^{\circ} \pm 0.14^{\circ}$ & $1.80^{\circ} \pm 1.09^{\circ}$ \\
& Azimuth & $0.18^{\circ} \pm 0.06^{\circ}$ & $1.06^{\circ} \pm 0.57^{\circ}$ \\
\hline \hline MOTION & Roll & $0.33^{\circ} \pm 0.10^{\circ}$ & $1.55^{\circ} \pm 0.80^{\circ}$ \\
& Elevation & $0.58^{\circ} \pm 0.15^{\circ}$ & $2.24^{\circ} \pm 0.50^{\circ}$ \\
& Azimuth & $0.40^{\circ} \pm 0.06^{\circ}$ & $1.98^{\circ} \pm 0.49^{\circ}$ \\
\hline
\end{tabular}

deviations of the OMD data from the best-fit straight lines were less than $0.07^{\circ}$ for all angles $\left( \pm 4^{\circ}\right)$ and less than 0.2 $\mathrm{mm}$ for the positions $( \pm 2-3 \mathrm{~cm})$. The rms deviations from the actual input values were less than $0.3^{\circ}$ and $0.3 \mathrm{~mm}$ for the angles and positions, respectively.

The measured changes in the other outputs for variations in one input (crosstalk) are shown in Fig. 7 and Table II. Except at the extreme values of the inputs, which are unlikely to be seen in practice, angular crosstalk is typically $<0.1 \%$ deg of rotational input and $<0.3 \% \mathrm{~cm}$ of translational input. Position crosstalk for a translational input is $<0.5 \mathrm{~mm} / \mathrm{cm}$ of input. Since typical angular head motions are $1-2^{\circ}$ and translational motions are 1-2 $\mathrm{mm}$ [1], the effect of this crosstalk is negligible.

\section{Accuracy in Subjects}

Fig. 8 shows typical angular motions as measured in one subject by both the OMD and the Spacetracker. It can be seen that the OMD follows the Spacetracker well during the entire study, even during periods of relatively large angular movements.

Table III shows the average rms and maximum absolute differences between the two sets of motion measurements from the OMD and the Spacetracker, averaged over the four studies (two subjects, two sessions each). The results indicate that all angles are within $0.2-0.6^{\circ} \mathrm{rms}$ between the two motiondetection systems for all parts of the study.

\section{DISCUSSION}

The OMD system described in this paper meets all of the design objectives we had established for a device to be useful for measuring head motion inside an ECT scanner. It has the requisite accuracy, linearity, resolution, range, stability, and data rate. It has been shown to work well on subjects with different head sizes and to be accurate when operated inside a PET scanner. While the spike correction, found necessary in the studies on subjects at times of abrupt and large changes in orientation, effectively reduces the sampling rate below 1 $\mathrm{Hz}$, such spikes were infrequent and occurred primarily during periods of rapid, large motion changes. When subjects lay quietly, as will be the case for most PET studies, no spikes were observed, and the sampling rate was, in fact, better than $1 \mathrm{~Hz}$. However, the circuitry that controls the lamps and is the 

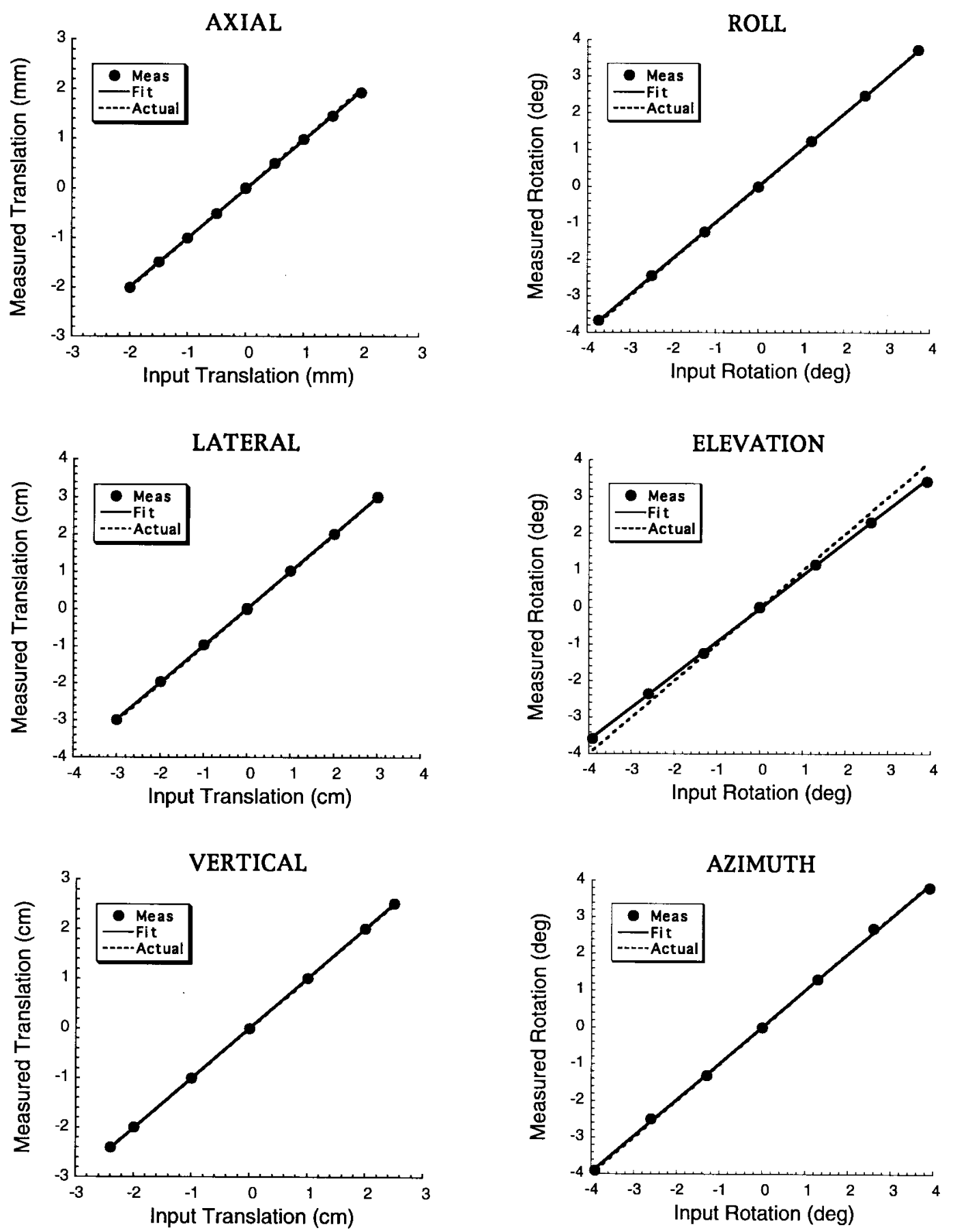

Fig. 6. Linearity and accuracy of the OMD. The measured OMD results are shown for known translational or rotational inputs from a mechanical test fixture with the triad located inside the scanner bore. The data points are the measured data for inputs in all six DOF's. The solid line represents the best-fit line to the data; the dashed line is the line of identity.

main reason for the 0.7-s sampling period, could be optimized to improve the sampling rate and further reduce the likelihood of spikes' occurring.

The system is designed to be nominally symmetric. It has been dismantled and setup numerous times without extraordinary means being taken to ensure this condition. Except for small variations in the crosstalk, the nominal performance has been consistent with that presented here. With some modifications to (1), the system could be adapted for use in a nonsymmetric geometry.
The short-term variability of the OMD is acceptably small. Noise would not be expected to impact any measure of head motion significantly. The system was also shown to be quite stable over time. The deviations observed were likely due in part to vibrations of the detector's test mounting fixture and the lamp triad (e.g., by slamming of nearby doors).

During initial performance characterization of the system, it was seen that stray light reflected from adjacent surfaces can cause subtle artifacts. For example, when care was not taken to minimize reflections, a pure azimuth input sometimes resulted 

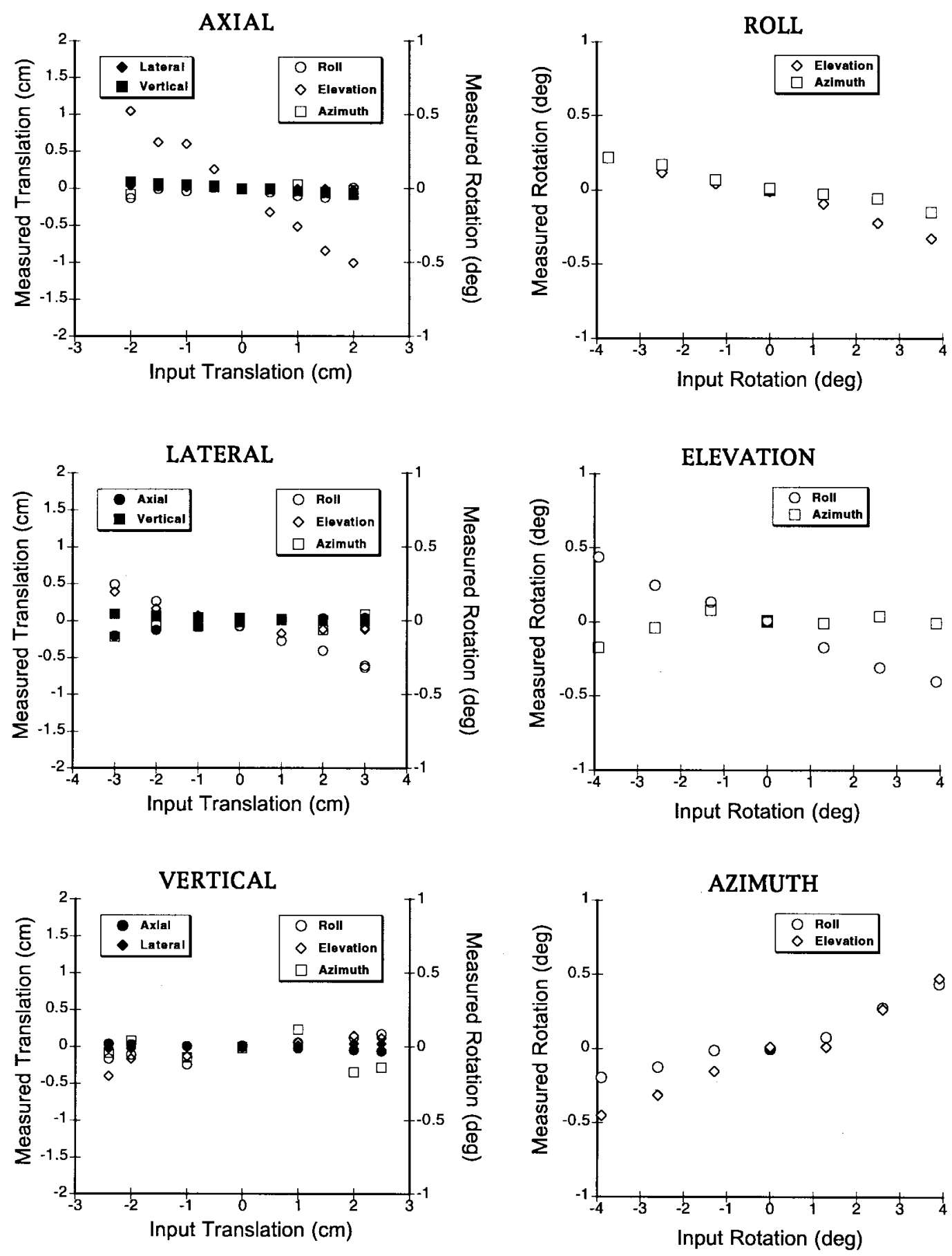

Fig. 7. Crosstalk of the OMD. The measured OMD results for the other DOF's not being changed are shown for the known translational and rotational inputs from the mechanical test fixture. Translational crosstalk for angular inputs are not shown, since the lamps would also translate with a rotation, depending on the (unknown) distance between the lamps and the axes of rotation. Typical angular head motions are $1-2^{\circ}$; translational motions are on the order of 1-2 mm [1].

in a measured roll of $\sim 10 \%$ of the azimuthal input value, as well as errors of $\sim 5 \%$ in the measured azimuth value. This problem was reduced by 1) recessing the light bulbs inside a tube that had small notches in the plane of the two sensor optical axes (see Section II); 2) appropriately placing black cloth on adjacent surfaces (e.g., inside the scanner bore); 3) employing the auxiliary lightshield shown in Fig. 2. All results presented in this paper were made with these modifications in place.
The results in Table I show that the system is linear for all variables, and except for elevation, the slopes are essentially unity. In addition, the deviation from unity for elevation could be corrected with a scale factor with limited impact on the accuracy of the roll results [see (2)], since the angular motions are typically small. Table II shows that crosstalk, while negligible for the ranges of inputs of interest, nevertheless exists to some extent. We believe that the reason for these two departures from ideal behavior is residual effects of reflected 

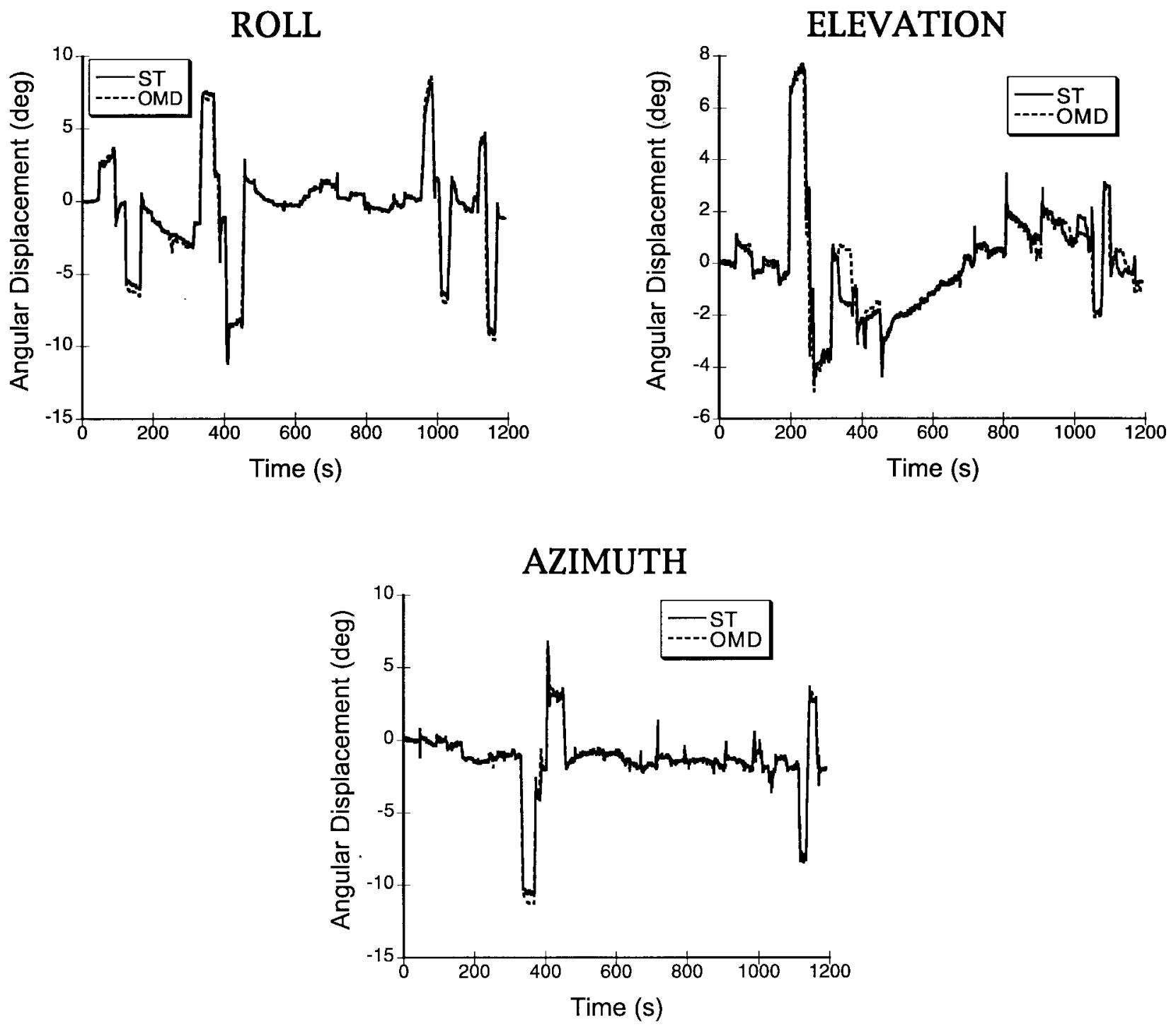

Fig. 8. Typical angular motions as measured in a subject by both the OMD and the Spacetracker (ST). The two devices measured the angular motions simultaneously as the subject executed a series of controlled angular motions (0-450 s), then lay quietly (450-950 s), and then carried out more controlled angular motions (950 s-end). The results shown are after correction for spikes in both Spacetracker and OMD data sets at times of abrupt motion changes.

stray light. (The light reflections off the curve of the scanner bore can introduce a small coupling between lateral and vertical motions, for example.) When the same tests were done outside the scanner bore, all slopes were within $2 \%$ of unity, and crosstalk was essentially zero $(<0.02 \mathrm{~mm} / \mathrm{cm},<0.03 \% \mathrm{~cm}$, and $<0.03^{\circ} / \mathrm{deg}$ ) for all DOF's except for roll for vertical and azimuth inputs $\left(0.04 \% \mathrm{~cm}\right.$ and $0.08^{\circ} / \mathrm{deg}$, respectively). For the above-described tests inside the scanner bore, we were unable to treat the bore surface by the ordinary techniques to eliminate reflections (e.g., apply optical-flat black paint, sand surface, etc.). We intend to do these prior to subsequent studies and are confident that these measures will eliminate the remaining undesirable effects by making the optical situation equivalent to that outside the scanner.

Another way to eliminate these problems would be to use light-emitting diodes (LED's), which are more directional than the incandescent lamps used. However, the light source must have an illumination angle that exceeds the angle between the lines joining the triad and the two detectors $\left(2 \alpha=77^{\circ}\right.$, see Fig. 1). Since even wide-angle LED's have a considerably smaller field of illumination, this requires each "point" to consist of a pair of LED's, each oriented toward a detector. This configuration was tried, but the lack of position coincidence of the two LED's resulted in errors that compromised the system accuracy unless further correction factors would be incorporated in the algorithm. For high and variable ambient light levels, high-power infrared or visible LED's, combined with spectral filtering and/or pulsing and synchronous detection, would be needed. Since typical ambient light levels in ECT can be maintained sufficiently low without requiring significant eye accommodation that these temporal and spectral techniques are not necessary, we chose to avoid the associated complexities of LED's by using the miniature tungsten lamps and minimizing reflections as described above.

Another area in which we initially chose to avoid complexity was in not using minimization methods to utilize 
redundant information about position and orientation from all three lamps. Because the triad is essentially a rigid structure, it did not seem necessary to use more than one lamp to describe its translation or to use more than two lamps to determine azimuth and elevation. Due to the small values of these two angles, their effect on the measurement of roll [see (2)] is negligible. This expectation has been validated by the accuracy of the motion measurements, although the algorithms could be easily modified, if necessary.

The rms differences in the studies of accuracy in subjects indicate that the roll and azimuth measurements agree well between the two systems. The deviations for the elevation angles are somewhat larger, although still acceptably small. The reproducibility of the OMD in the same subject for two measurement sessions and the accuracy of the device for subjects with different head sizes were both high, as reflected in the small standard deviation of the rms values.

In the measurement of head motion, it is assumed that each motion detection device is rigidly attached to the subject's head. The comparison between the OMD and the Spacetracker involved different methods of attaching the two motion detection systems to the head. The OMD used plastic balls in the openings of the ears with stabilizing attachments at the temples, while the Spacetracker used a biteplate held firmly in place by the subjects. Both of these attachment schemes can be expected to fail under certain circumstances. In particular, a frown can move the OMD even in the absence of any real head motion and, if held for an extended period, can lead to an erroneous measurement of motion. Likewise, the Spacetracker will measure an apparent head motion if the subject fails to maintain firm pressure on the biteplate. In the plot of elevation in Fig. 8, the discrepancy between the two devices at $\sim 350 \mathrm{~s}$ is most likely due to the latter problem.

The choice of lens focal length is a tradeoff between illumination level and error due to lens geometric distortion. A longer focal length results in more light at the sensor, which leads to lower noise levels and better resolution. However, use of the edges of the field reduces the positional accuracy. This is exacerbated if the legs of the triad are too long. The original $10-\mathrm{cm}$ triad leg length was reduced to $7.6 \mathrm{~cm}$ for this reason without significantly compromising the angular accuracy. This also enabled the working volume to be maintained at a level that better allows accommodation of different sized subjects as well as relatively large motions.

Studies validating the application of motion correction using the external data measured with the OMD to patient images are currently underway.

\section{ACKNOWLEDGMENT}

The authors gratefully acknowledge J. Sullivan, Chief, and P. Fitz of the Precision Instrument Shop, Biomedical Engineering and Instrumentation Program, NCRR, NIH for their fine work in fabricating the light fixture assembly.

\section{REFERENCES}

[1] M. V. Green, J. Seidel, S. D. Stein, T. E. Tedder, K. M. Kempner, C. Kertzman, and T. A. Zeffiro, "Head movement in normal subjects during simulated PET brain imaging with and without head restraint," J. Nucl. Med., vol. 35, pp. 1538-1546, 1994.

[2] U. E. Ruttimann, P. J. Andreason, and D. Rio, "Head motion during positron emission tomography: Is it significant?," Psych. Res: Neuroimag., vol. 61, pp. 43-51, 1995.

[3] R. A. Koeppe, V. A. Holthoff, K. A. Frey, M. R. Kilbourn, and D. E. Kuhl, "Compartmental analysis of [11C] flumazenil kinetics for the estimation of ligand transport rate and receptor distribution using positron emission tomography," J. Cereb. Blood Flow Metab., vol. 11, pp. 735-744, 1991.

[4] A. Minoshima, K. L. Berger, K. S. Lee, and M. A. Mintun, "An automated method for rotational correction and centering of threedimensional functional brain images," J. Nucl. Med., vol. 33, pp. 1579-1585, 1992.

[5] R. R. Fulton, B. F. Hutton, M. Braun, B. Ardekani, and R. Larkin, "Use of 3-D reconstruction to correct for patient motion in SPECT," Phys. Med. Biol., vol. 39, pp. 563-574, 1994.

[6] J. Pavia, D. Ros, A. M. Catafau, F. J. Lomena, M. Huguet, and J. Setoain, "3-dimensional realignment of activation brain single-photon emission tomographic studies," Eur. J. Nucl. Med., vol. 21, pp. 1298-1302, 1994.

[7] R. P. Woods, S. R. Cherry, and J. C. Mazziotta, "Rapid automated algorithm for aligning and reslicing PET images," J. Comput. Assist. Tomogr., vol. 16, pp. 620-633, 1992.

[8] A. Dubey, J. Edgeworth, W. L. Rogers, and N. H. Clinthorne, "A patient motion correction system for tomographic imaging based on CCD video cameras," J. Nucl. Med., vol. 34, p. 90P, 1993.

[9] Y. Picard and C. J. Thompson, "Digitized video subject positioning and surveillance system for PET," Trans. Nucl. Sci., vol. 42, pp. 1024-1029, 1995.

[10] M. Menke, M. S. Atkins, and K. R. Buckley, "Compensation methods for head motion detected during PET imaging," IEEE Trans. Nucl. Sci., vol. 43, pp. 310-317, 1996.

[11] H. Goldstein, Classical Mechanics, 2nd ed. Reading, MA: AddisonWesley, 1981, p. 609. 